\title{
Measurement of Wave Attenuation in Buried Plastic Water Distribution Pipes
}

\author{
Fabrício César Lobato de Almeida ${ }^{1, *}$ - Michael John Brennan ${ }^{1}$ - Phillip Frederick Joseph ${ }^{2}$ - \\ Simon Dray ${ }^{3}$ - Stuart Whitfield ${ }^{4}$ - Amarildo Tabone Paschoalini ${ }^{1}$ \\ ${ }^{1}$ University Estadual Paulista, Department of Mechanical Engineering, Brazil \\ 2 University of Southampton, Institute of Sound and Vibration Research, United Kingdom \\ ${ }^{3}$ Hydrosave, United Kingdom \\ 4 South Staffs Water, United Kingdom
}

\begin{abstract}
Leaks in pipes are a common issue encountered in the water industry. Acoustic methods are generally successful in finding and locating leaks in metallic pipes, however, they are less effective when applied to plastic pipes. This is because leak-noise signals are heavily attenuated due to high damping in the pipe-wall and sound radiation into the soil. As result, high frequency leak noise does not travel long distances. To determine how far leak noise may travel in a pipe at any frequency, the attenuation of the wave responsible for leak noise propagation should be known. In this paper a new method to estimate this is described. The method is then applied to some measurements made on a bespoke pipe-test rig in the UK, and the results are compared with theoretical predictions.
\end{abstract}

Keywords: leak detection, wave attenuation, water industry, plastic pipes

\section{INTRODUCTION}

Water distributions systems are susceptible to leakage, which results in a substantial wastage of water. The social and environmental effects due to leakage problems are also a matter of concern. Recently, a survey about the costs of installation/repair work of buried infrastructure in the UK has estimated that street works cost about $£ 7 \mathrm{bn}$ in losses for the government annually; Social costs account for about $£ 5.5 \mathrm{bn}$ and damage costs are about $£ 1.5 \mathrm{bn}$ [1].

Water loss in distribution systems can typically reach between 20 and $30 \%$ of the total water production [2], but in some extreme cases this figure can rise up to $50 \%$ [3]. Leakage is the main issue responsible for such loss. Energy to supply water is one of major costs in developing countries, and it may easily consume $50 \%$ of a municipality's budget in the developing world [4]. Hence, reducing wastage of water through leaks directy affects the cost of water distribution. Furthermore, it is estimated that between 2 and 3\% of the world's energy consumption is used to pump and treat water for urban and industrial purposes [4]. Approximately 30 to $50 \%$ of water is lost globally due to water leakage.

The noise from a leak in a buried plastic pipe generally has low frequency content, occurring well below the pipe ring frequency [5]. In this frequency region, only four types of waves are, in general, responsible for most of the energy transfer in a pipe [6] and [7]. However, in buried plastic water pipes leak noise propagates in an axis-symmetric wave that is predominantly a fluid wave, but is strongly coupled to the pipe-wall, such that there is significant radial motion [5] and [7]. This strong coupling means that leak noise energy is dissipated within the pipe-wall and also radiates as sound into the ground [8] and [9]. The result is that the pipe acts as a low-pass filter [10], and hence measured leak noise tends to be below about $200 \mathrm{~Hz}$.

There are different ways of estimating the wave attenuation in pipes. Muggleton et al. [5], [8] and [11] have derived analytical expressions to predict the wavenumber in buried and submerged pipes for leak detection problems. The wavenumber is a frequency dependent complex quantity, the real part of which is related to the wavespeed, and imaginary part is related to the attenuation factor, which determines how far a wave can propagate along the pipe at any particular frequency. Hence, if the wavenumber can be predicted then the wave attenuation factor can be estimated. Measurements have also been carried out to determine the wavenumber in buried pipes [9], [12] and [13].

The aim of this paper is to investigate a new technique to estimate the wave attenuation factor insitu, in buried pipes. This technique is based on the envelope of the cross-correlation function between two vibration signals measured on the pipe, either side of the leak. An advantage of the method is that it can also be used in the presence of a leak, which could potentially affect the estimate of wave attenuation. Following a description of the method in the next section, and some simulations in Section 2, some experiments on a bespoke test rig are reported in Section 3 to validate the proposed method. 


\section{THEORETICAL DEVELOPMENT}

Fig. 1 shows a schematic of a pipe with a leak between measurement Positions 1 and 2, together with actuators and sensors attached to each measurement position. The measurement positions are at convenient access points.

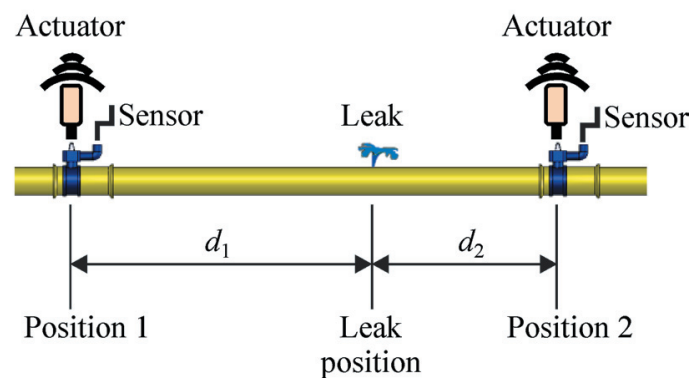

Fig. 1. Schematic of a buried pipe showing the sensors and actuators mounted at measurement Positions 1 and 2 with the leak located between these positions

Two measurements are carried out. First, the actuator at Position 2 is excited, generating a wave which propages along the pipe to Position 1 . The vibration of the pipe is measured using two sensors, one mounted next to the actuator at Position 2 and the other mounted at Position 1. If there is coherence between the two measured signals, the crosscorrelation function can be calculated [14], and give information about the wavespeed and attenuation factor. The actuator at Position 1 is then excited, and the measurement procedure is repeated. The wave then propagates in the opposite direction. The procedure to calculate the wave attenuation in $\mathrm{dB} / \mathrm{m}$ for the section of the pipe involves some manipulation of the crosscorrelation functions, including the determination of their envelopes using the Hilbert transform [15]. Moreover, an estimation of the maximum distance that the leak noise would travel at each frequency can be calculated.

In the presence of a leak two types of excitation act simultaneously on the pipe. One is the controlled excitation from an actuator, and the other is from the leak. The frequency response function (FRF) between the pressure at the leak position and the acceleration on the pipe at a distance $x$ from the leak is given by [10]:

$$
H_{a}(\omega, x)=-\omega^{2} H(\omega, x)
$$

where

$$
H(\omega, x)=e^{-\omega \beta x} e^{-i \omega x / c},
$$

in which $\beta$ and $c$ are the attenuation factor and the phase velocity of the propagating wave, respectively. The frequency response function between the two accelerometer signals is given by $H\left(\omega, d_{1}+d_{2}\right)$. In Eq. (2) $-\omega \beta$ is the imaginary part of the wavenumber $\operatorname{Im}\{k\}$, which has units of $1 / m$, and the attenuation factor in the pipe is given by $20 \operatorname{Im}\{k\} / \ln \{10\}$. If the actuator at Position 2 is driven at the same time as there is a leak, the cross-spectral density function $S_{21}(\omega)$ between the measurement Positions 2 and 1, is given by:

$$
\begin{aligned}
S_{21}^{(2)}(\omega)= & \omega^{4} H^{*}\left(\omega, d_{1}\right) H\left(\omega, d_{2}\right) S_{l l}(\omega)+ \\
& +H\left(\omega, d_{1}+d_{2}\right) S_{e e}(\omega),
\end{aligned}
$$

where $S_{l l}(\omega)$ and $S_{e e}(\omega)$ are the auto-spectral density functions of the leak and the acceleration at the actuator position, respectively, and the superscript (2) indicates that the actuator at Position 2 is being excited. The corresponding cross-correlation function $R_{21}^{(2)}(\tau)$, can be determined from the inverse Fourier transform $F^{-1}$ of Eq. (3) to give $R_{21}^{(2)}(\tau)=F^{-1}\left\{S_{21}^{(2)}(\tau)\right\}$, which can be written as:

$$
\begin{aligned}
R_{21}^{(2)}(\tau)= & R_{l l}(\tau) \otimes h(\tau) \otimes \delta\left(\tau-T_{0}\right)+ \\
& +R_{e e}(\tau) \otimes \psi(\tau) \otimes \delta\left(\tau-T_{\text {act }}\right),
\end{aligned}
$$

where $\otimes$ denotes convolution, $R_{l l}(\tau)=F^{-1}\left\{S_{l l}(\omega)\right\}$ is the auto-correlation of the leak signal, $R_{e e}(\tau)=$ $F^{-1}\left\{S_{e e}(\omega)\right\}$ is the auto-correlation function of the acceleration signal at the actuator excitation position, $\psi(\tau)=F^{-1}\left\{e^{-\omega \beta\left(d_{1}+d_{2}\right)}\right\}, h(\tau)=F^{-1}\left\{\omega^{4} e^{-\omega \beta\left(d_{1}+d_{2}\right)}\right\}$, $T_{0}=\left(d_{1}-d_{2}\right) / c$ is the time delay due to the leak, $T_{\text {act }}=\left(d_{1}+d_{2}\right) / c$ is the time delay due to the actuator excitation and $\delta()$ is the Dirac delta function. Eq. (4) shows that the delta function $\delta\left(\tau-T_{0}\right)$ is smeared by the leak spectrum $S_{l l}(\omega)$ and the behaviour of $\omega^{4} e^{-\omega \beta\left(d_{1}+d_{2}\right)}$, and the delta function $\delta\left(\tau-T_{\text {act }}\right)$ is smeared by the external excitation spectrum $S_{e e}(\omega)$ and the frequency characteristics of $\Psi(\omega)=e^{-\omega \beta\left(d_{1}+d_{2}\right)}$.

For simplicity, in the model the spectral characteristics of the leak and the excitation are considered to be white noise. The auto-spectral density function of the leak is thus assumed to be given by $S_{l l}(\omega)=S_{0}$ and the external excitation by $S_{e e}(\omega)=n S_{0}$, where $n$ is the ratio of the spectral density of the leak noise to the spectral density of the external excitation. If the signals from the sensors are passed through ideal band-pass filters with frequency response $G(\omega)$, in which $G(\omega)=1$ for $\omega_{\text {low }} \leq \omega \leq \omega_{\text {upp }}$ and zero otherwise, the cross-correlation function becomes: 


$$
\begin{aligned}
R_{21}^{(2)}(\tau)= & S_{0}\left[h(\tau) \otimes g(\tau) \otimes \delta\left(\tau-T_{0}\right)+\right. \\
& \left.+n \psi(\tau) \otimes \mathrm{g}(\tau) \otimes \delta\left(\tau-T_{\text {act }}\right)\right],
\end{aligned}
$$

where $g(\tau)$ is given by $g(\tau)=F^{-1}\{G(\omega)\}$, which can be written as:

$$
g(\tau)=\frac{\Delta \omega}{\pi} \frac{\sin (\Delta \omega \tau / 2)}{\Delta \omega \tau / 2} \cos \left(\omega_{\operatorname{cen}} \tau\right)
$$

in which $\Delta \omega=\omega_{\text {upp }}-\omega_{\text {low }}$ is the bandwidth of the filter and $\omega_{\text {cen }}=\left(\omega_{\text {low }}+\omega_{\text {upp }}\right) / 2$ is the central frequency. The band-pass filter introduces a ripple with frequency $\omega_{\text {cen }}$ into the cross-correlation function. Eq. (5) shows that the delta function is further smeared by the introduction of the band-pass filter. According to Eq. (5) there are two possible peaks present in the crosscorrelation function; one is due to the leak and another is due to the external excitation. In this particular case, where the external excitation source is at Position 2, the peak in the cross correlation function related to the external excitation occurs at $\tau=\left(d_{1}+d_{2}\right) / c$. However, when the external excitation is at Position 1, the peak occurs in $R_{21}^{(2)}(\tau)$ at $\tau=-\left(d_{1}+d_{2}\right) / c$. The peak in the cross-correlation function due to the leak occurs at $\tau=\left(d_{1}-d_{2}\right) / c$ irrespective of the position of the external source.

If the band-pass filter is broad enough [10] such that $\Psi(\omega)<<1$, the envelope of the cross correlation function $R_{21}^{(2)}(\tau)$ or $R_{21}^{(1)}(\tau)$ normalised by its peak value when $\tau=T_{\text {act }}$, is given by [10]:

$$
\operatorname{Env}\left(R_{21}^{(2)}(\tau)\right)=\operatorname{Env}\left(R_{12}^{(1)}(\tau)\right)=\frac{\beta d}{\sqrt{(\beta d)^{2}+\left(\tau-T_{\text {act }}\right)^{2}}} .
$$

The product of the normalised envelopes can be shifted so that its peak occurs at 0 rather than $T_{\text {act }}$, i.e.,

$$
\operatorname{Env}\left(R_{21}^{(2)}\left(\tau+T_{\text {act }}\right)\right) \times \operatorname{Env}\left(R_{12}^{(1)}\left(\tau+T_{\text {act }}\right)\right)=\frac{1}{1+(\tau / \beta d)^{2}} .
$$

It can be seen that the envelope given by Eq. (8) is a function of the attenuation factor, which is the unknown quantity to be determined, $d$ which is known and $\tau$ which can be obtained from the measurements. Two ways of estimating the attenuation factor are proposed here. The first is based on setting Eq. (8) to be equal to $1 / 2$, which is a measurable quantity. In this case the attenuation factor is given by:

$$
\beta=\frac{\left|\tau_{1 / 2}\right|}{d},
$$

where $\left|\tau_{1 / 2}\right|$ is the absolute value of $\tau$ when Eq. (8) is equal to $1 / 2$. The second method is based on the area $A$ of the envelope given in Eq. (8). If it is assumed that the envelope is symmetric then:

$$
A=2 \int_{0}^{\tau_{1}} \frac{1}{1+(\tau / \beta d)^{2}} d \tau
$$

where $\tau_{1}$ is the upper limit of the integral. Evaluating the integral in Eq. (10) results in:

$$
\beta=\left.\frac{A}{2 d \arctan (\tau / \beta d)}\right|_{0} ^{\tau_{1}} .
$$

Note that if $\tau_{1} \rightarrow \infty$ then $\arctan (\tau / \beta d) \rightarrow \pi / 2$, so that $\beta=A / d \pi$. In practice, the area $\mathrm{A}$ is calculated numerically from measured results and in Eq. (11) $\tau_{1}$ is set to a finite value to avoid noise in the data.

\section{SIMULATIONS}

To illustrate the methodology described in the previous section, some simulations are carried out prior to presenting some experimental results in the following section. The system in Fig. 1 is considered with the parameters given in Table 1, which corresponds to one of the measurements made in the Blithfield test rig, which is described in Section 3.

Table 1. Parameters used to illustrate the method to determine the wave attenuation factor

\begin{tabular}{cc}
\hline$d_{1}$ & $30 \mathrm{~m}$ \\
\hline$d_{2}$ & $20 \mathrm{~m}$ \\
\hline$\beta$ & $2.9 \times 10^{-4} \mathrm{~s} / \mathrm{m}$ \\
\hline$c$ & $390 \mathrm{~m} / \mathrm{s}$ \\
\hline$f_{\text {low }}=\omega_{\text {low }} / 2 \pi$ & $20 \mathrm{~Hz}$ \\
\hline$f_{\text {upp }}=\omega_{\text {upp }} / 2 \pi$ & $35 \mathrm{~Hz}, 200 \mathrm{~Hz}$ \\
\hline$n$ & $2 \times 1010$ \\
\hline
\end{tabular}

Note that it is assumed arbitrarily that the amplitude of the peak due to the leak is less than half of the amplitude of the peak due to the actuator. This assumption is based on the experience that the actuator excitation has always dominated the signal at one of the sensors in the experiments carried out by the authors.

Two cases are considered. In the first case, a large bandwidth is set with lower and upper limits set to 20 and $200 \mathrm{~Hz}$, respectively. This represents a case where the vibration due to the actuators is effectively transmitted along the pipe, leading to good coherence over a wide frequency range. Second, a much smaller 
bandwidth is set with lower and upper limits set to 20 and $35 \mathrm{~Hz}$, respectively. This represents a case when the distance and or damping in the pipe is large enough to significantly attenuate high frequency vibration at the sensor positions.

Figs. 2a(i) and (ii) show, $R_{21}^{(2)}(\tau)$ and $R_{21}^{(1)}(\tau)$ normalized by their maximum values together with their envelopes calculated using the Hilbert transform, for the two different bandwidths. The largest peaks in each of the cross-correlation functions correspond to the time delays due to wave propagation from Positions 2 to 1 , and from Positions 1 to 2 . Note that one of these occurs when $\tau$ is negative, and one occurs when $\tau$ is positive. The small peak in Fig. 2a(i) is due to the leak.

The effect of a much reduced bandwidth on the appearance of the peak due to the leak can be seen in Fig. 2a(ii). The peak cannot be seen as it is masked by side-lobes of the peaks from the actuator excitation. This effect can be understood using Eqs. (5) and (6). When the filter bandwidth decreases, the side-lobes around the main peaks in the envelopes increase, and move away from these peaks as shown in Fig. 2a(ii).
A rule of thumb as to whether or not the peak due to the leak is masked, can be determined using an approximation for the envelopes, which is given by the modulus of the sinc function in Eq. (6). It is found that the peak due to the leak will be masked and not be present in the envelopes if $T_{0}<T_{\text {act }}-6 \pi / \Delta \omega$.

To simplify the presentation of the data from the two measurements discussed above, they are combined as follows. First, $R_{12}^{(1)}(\tau)$ is calculated instead of $R_{21}^{(1)}(\tau)$, which has a peak for positive $\tau$ instead of negative $\tau$. If the wave speed estimate is the same in each direction then this coincides with the peak for $R_{21}^{(2)}(\tau)$. The product of the envelopes of these cross-correlation functions is then calculated. This has a large peak corresponding to the time it takes for a wave to propagate from the actuator to the sensor, and the peak corresponding to the leak is much diminished. This can be seen in Figs. 2b(i) and (ii), which are normalised plots of the product of the envelopes of $R_{12}^{(1)}(\tau)$ and $R_{21}^{(2)}(\tau)$, for two different bandwidths. It can be seen that the effect of multiplying the two envelopes together is to accentuate the peak due to the actuators and diminish
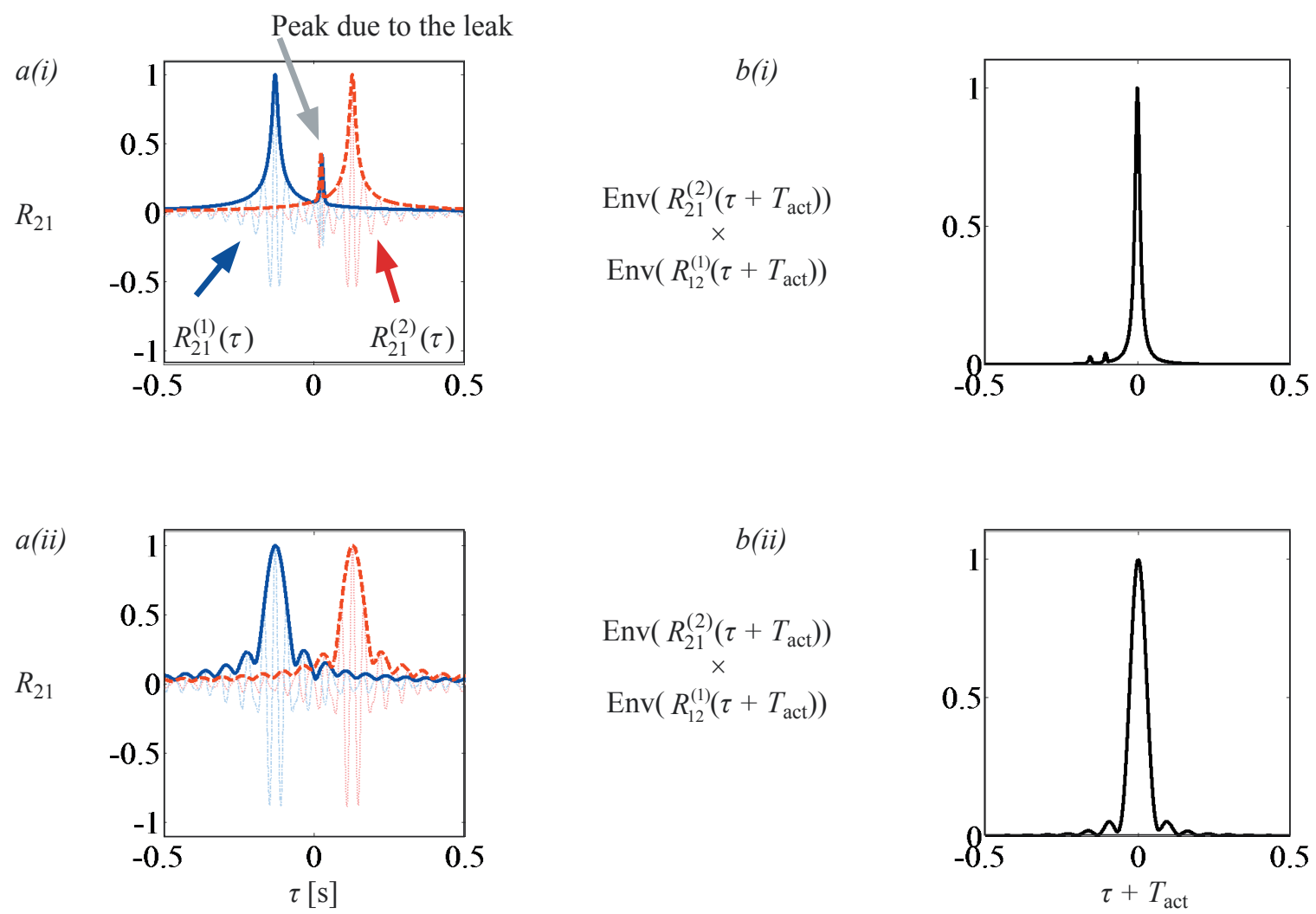

Fig. 2. Simulations illustrating the technique to isolate the effects of the presence of a leak; the labels 'i' and 'ii' correspond to when the lower and upper limits of the band-pass filter are set at 20 to $200 \mathrm{~Hz}$ and 20 to $35 \mathrm{~Hz}$, respectively; a(i) and a(ii), the envelope of the crosscorrelation function $R_{21}$ given by Eq. (5); b(i) and b(ii), the product of the envelopes of $R_{12}^{(1)}\left(\tau+T_{\text {act }}\right)$ and $R_{21}^{(2)}\left(\tau+T_{\text {act }}\right)$ 
the peak due to the leak (although there are now two small peaks for a large bandwidth, as seen in Fig. 2b(i)). When the bandwidth is small, as in Fig $2 b(i i)$ only a single peak due to the excitation by the actuators is evident, together with some small sidelobes. Further processing of the data to determine the wave attenuation from the product of the envelopes is discussed in the next section.

\section{EXPERIMENTAL WORK}

The pipe rig used in this work is located at the Blithfield reservoir in Staffordshire, UK. Fig. 3 shows a schematic side-view highlighting the access points. The pipe is buried in an open field close to the South Staffs Water reservoir facility. The pipe is made from high performance polyethylene (HPPE) with Young's modulus of $1 \times 10^{9} \mathrm{~N} / \mathrm{m}^{2}$. The pipe rig is 120 $\mathrm{m}$ long, consisting of section lengths of 10, 20 and 30 metres, which have an outer radius of $80 \mathrm{~mm}$ and wall thickness of $9.85 \mathrm{~mm}$. There are 6 access points as shown in Fig. 3.

The pipe extremity close to Position 1 is connected to the mains water distribution pipe, which supplies water at a pressure of about 6 bar. At the other extremity the pipe is terminated with a blank. All the access points are set in concrete to provide a rigid support for the pipe connections, while the pipe sections are buried in the ground at a depth of about $0.8 \mathrm{~m}$. Fig. $4 \mathrm{a}$ shows one of the access points.

Leak noise could be generated by opening a secondary valve fitted to a blanking piece attached to the hydrant as shown in the sketches in Figs. $4 \mathrm{~b}$ and c. A pressure gauge was also attached to the hydrant as shown in Fig. 4c

The method described Section 1 was implemented using experimental data from the Blithfield test rig. As mentioned previously, sensors and actuators were mounted at Positions 1 and 2 and the leak was induced between these positions. Details of the transducers and instrumentation are given in Tab. 2. Fig. 5 shows

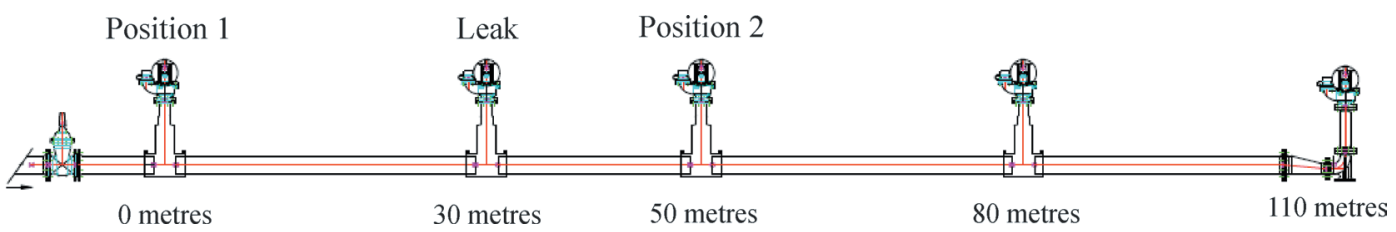

Fig. 3. Schematic of the pipe rig used in the experimental work, showing the distances between the access points, and the excitation/ measurement positions and the leak position
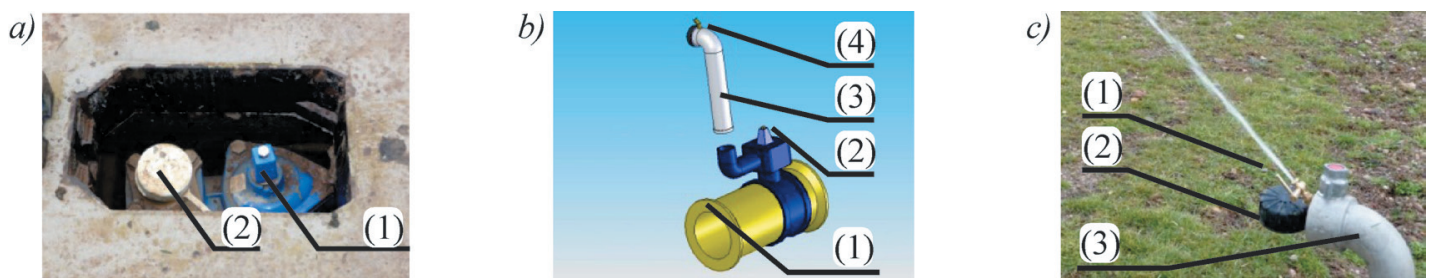

Fig. 4. Some components of the pipe test-rig; a) the pipe access point and its main valve: (1) Main valve; (2) connection point; b) sketch of the device used for generating the leak conditions: (1) plastic pipe; (2) main valve; (3) metal hydrant; (4) secondary valve; (c) pressure gauge and secondary valve attached to the hydrant: (1) secondary valve; (2) pressure gauge; (3) hydrant
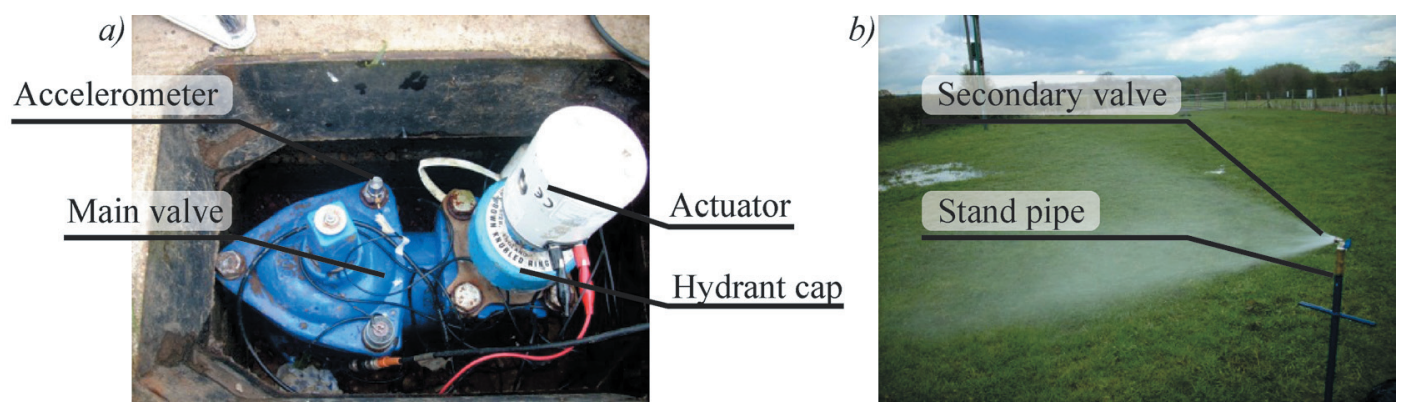

Fig. 5. Photograph of the test rig at Blithfield used to validate the methods to estimate the attenuation factor of a pipe in the presence of a leak; a) instrumentation at Positions 1 and 2; b) the leak induced in the pipe 
some photographs of the experimental set-up. Fig. 5a shows the instrumentation used at Positions 1 and 2, which are 50 metres apart, and Fig. 5 b shows the leak. The main valve shown in Fig. 5a allowed the water contained in the buried pipe to enter the hydrant. The leak was 30 metres away from Position 1.

The signals from the sensors were captured and digitised using a data acquisition system (DATS) made by PROSIG. This system is a 16 bit analog to digital converter (ADC) device type P5650, which has 8 synchronised input channels, and a sample rate of up to $100 \mathrm{kHz}$ per channel. The actuator was driven using a chirp signal between 20 and $200 \mathrm{~Hz}$, generated by a Hameg signal generator, reapeating every 3 seconds. The sampling frequency was $5 \mathrm{kHz}$, and a frequency resolution of $1 \mathrm{~Hz}$ was used in the subsequent spectral analysis.

Table 2. Measurement devices used in collection of the data

\begin{tabular}{lcc}
\hline Device & Manufacturer & Type \\
\hline Accelerometers & Bruel and Kjaer & 4383 and 4384 \\
\hline Shaker (actuator) & LDS & V201 \\
\hline Charge Amplifiers & Bruel and Kjaer & 2635 \\
\hline Function Generator & Hameg & HM8130 \\
\hline Acquisition System & Prosig & DATS \\
\hline
\end{tabular}

Fig. 6 shows the power-spectral density (PSD) of accelerometer-measured signals at Positions 1 and 2 and the coherence between these signals. Data are shown for simultaneous excitation of the actuator, and with leak excitation alone. The shaker was set at its highest level or turned off with the secondary valve fully open. It can be seen in Figs. $6 a$ and $b$ that the leak marginally dominates the signals measured at Position 1, but the actuator strongly dominates the signal at Position 2. It can also be seen that when the actuator is turned on, the coherence between the signals at Positions 1 and 2 changes significantly due to the interaction of the different sources of vibration.

Fig. 7a shows the cross-correlation coefficient between signals at Positions 1 and 2 for three cases; (a) when the pipe is excited at Position 2 by an actuator, (b) when the leak only excites the pipe, and (c) when both the leak and actuator act simultaneously to excite the pipe. Comparing Figs. 7a and $\mathrm{c}$ it can be observed that the peak in the cross-correlation coefficient reduces when the leak is turned on. This is because the leak marginally dominates the signal at Position 1 and the actuator strongly dominates the signal at Position 2 as shown in Fig. 6. Despite this, the largest peak in the cross-correlation coefficient corresponds to the
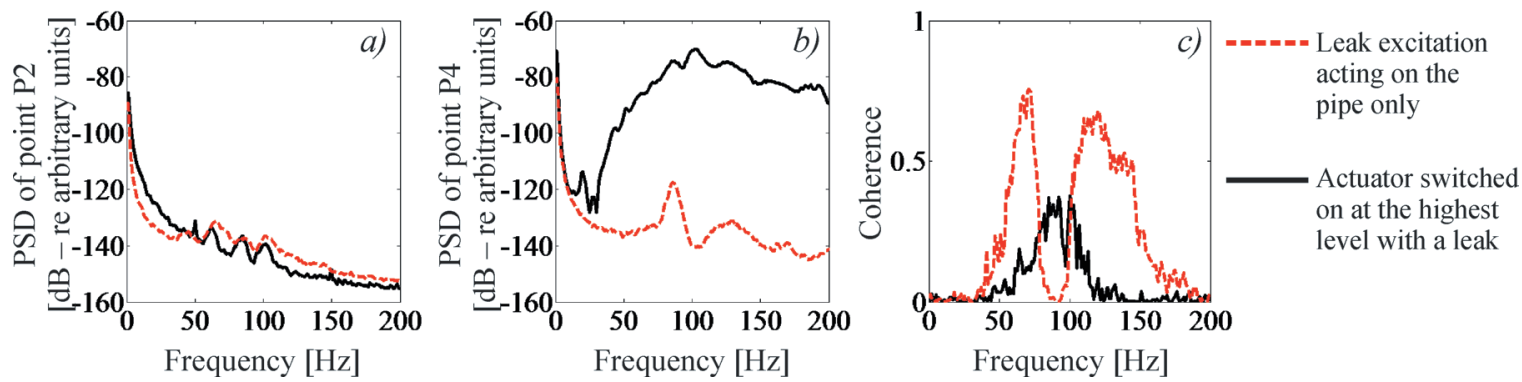

Fig. 6. Measurements at Positions 1 and 2 with the system being excited at Position 2; the leak was induced between Positions 1 and 2; a) PSD at Position 1; b) PSD at Position 2; c) the coherence between Positions 1 and 2
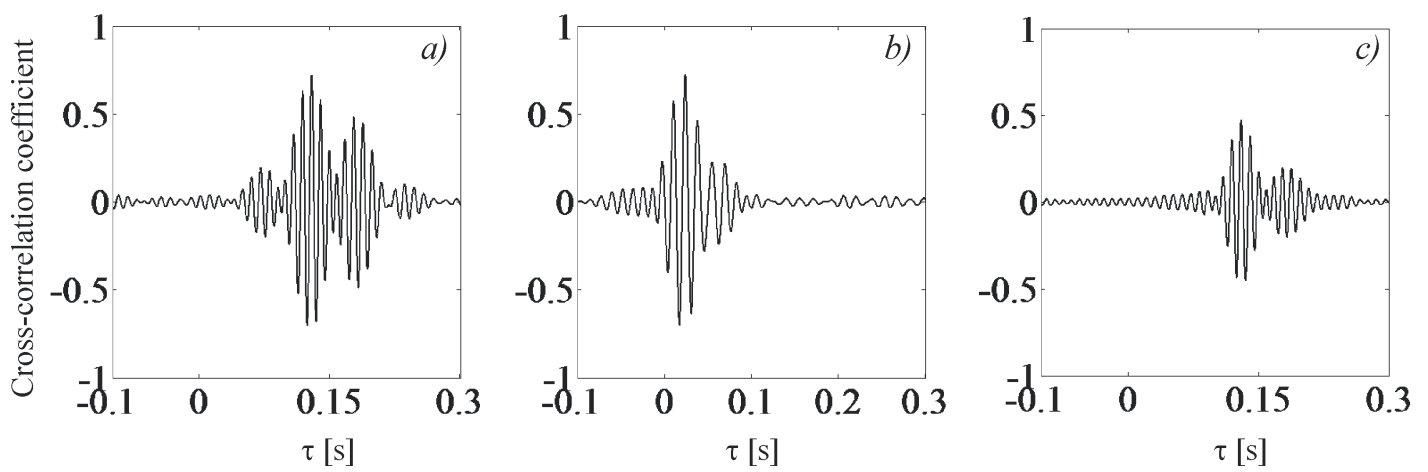

Fig. 7. The cross-correlation coefficient calculated from signals measured at Positions 1 and 2, when: a) the actuator is excited at Position 2 without any leak in the pipe; b) there is only a leak; and c) the actuator and leak acted simultaneously 
time delay due to the wave generated by the actuator as shown in Fig. 7c.

Following the calculation of the cross-correlation functions, their envelopes were determined as discussed in Section 1. Figures 8a and $\mathrm{b}$ show the results of the two techniques to estimate the attenuation factor discussed in the previous section. The experimental data is shown as a solid line and the envelopes given by Eq. (8) when the total loss is estimated by the method using Eq. (9) (red dashed line), and the other method using Eq. (11) (blue dashed-dotted line). The theoretical model only predicts the experimental result at time delays close to the peak in the product of the envelopes. Thus only experimental data close to the peak is taken into account in the calculation of the attenuation factor. This is determined by setting a threshold of 0.01 for the product of the envelopes and only data greater than this value around the peak is chosen. Fig. 8a shows the estimation of the attenuation factor (envelopes) with absence of a leak. Fig. 8b shows the estimation of the attenuation factor for a case with a leak present in the pipe.

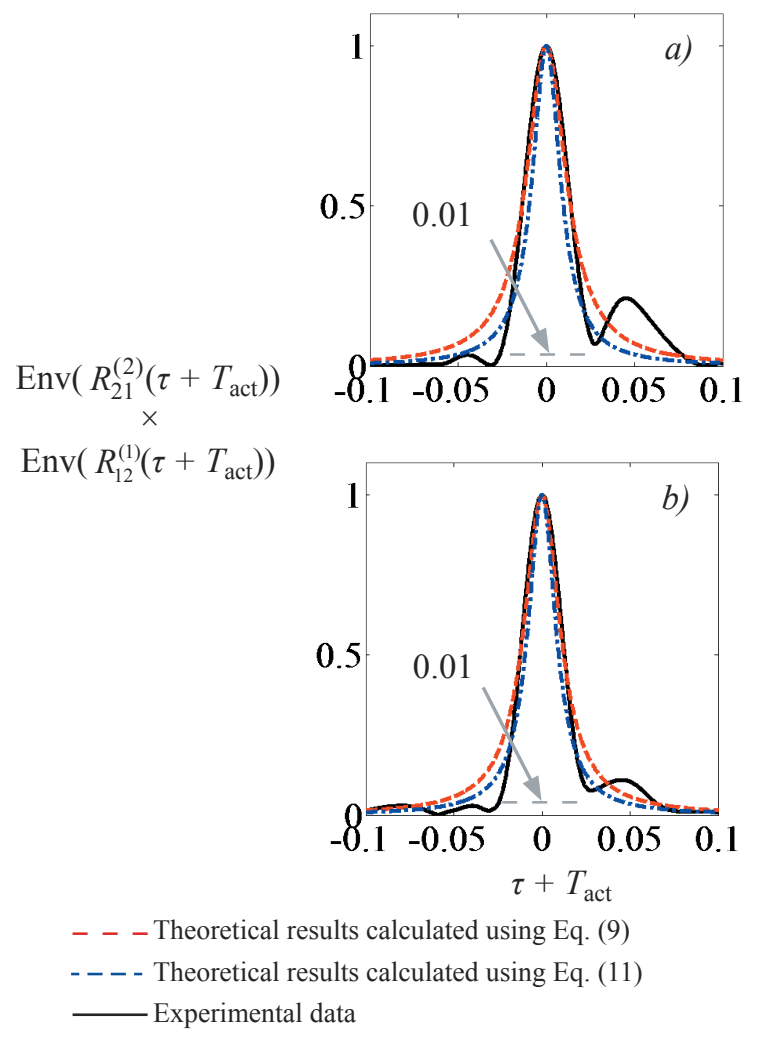

Fig. 8. The normalised product of the envelopes: a) no leak; b) with leak
With no leak, the attenuation factor was found to be $2.8 \times 10^{-4} \mathrm{~s} / \mathrm{m}$ and $1.9 \times 10^{-4} \mathrm{~s} / \mathrm{m}$ using Eqs. (9) and (11), respectively. When the leak was induced the attenuation factor was found to be $2.5 \times 10^{-4} \mathrm{~s} / \mathrm{m}$ and $1.9 \times 10^{-4} \mathrm{~s} / \mathrm{m}$ using Eqs. (9) and (11), respectively. The attenuation factors used to estimate the envelopes in Fig. 8 are sumarized in Table 3. The difference between the attenuation factor can be observed in Figs. $8 \mathrm{a}$ and $\mathrm{b}$ as the difference in shape between the envelopes. Thus the results show that the leak only has a marginal effect on the estimation of the attenuation factor.

Table 3. Attenuation factor calculated using Eq. (9) and Eq (11) for cases with and without a leak in the pipe

\begin{tabular}{ccc}
\hline Cases & $\begin{array}{c}\text { Attenuation factor } \\
\text { calculated using Eq. (9) }\end{array}$ & $\begin{array}{c}\text { Attenuation factor } \\
\text { calculated using Eq. (11) }\end{array}$ \\
\hline No leak & $2.8 \times 10^{-4} \mathrm{~s} / \mathrm{m}$ & $1.9 \times 10^{-4} \mathrm{~s} / \mathrm{m}$ \\
\hline Leak & $2.5 \times 10^{-4} \mathrm{~s} / \mathrm{m}$ & $1.9 \times 10^{-4} \mathrm{~s} / \mathrm{m}$ \\
\hline
\end{tabular}

The attenuation in the pipe at each frequency in $\mathrm{dB} / \mathrm{m}$ is given by [10]:

$$
\text { Attenuation }=8.67 \beta \omega \text {. }
$$

This can be determined from a measurement by [10]:

$$
\text { Attenuation }=\frac{-20 \ln \left|H\left(\omega, d_{1}+d_{2}\right)\right|}{\left(d_{1}+d_{2}\right) \ln (10)} \text {, }
$$

Thus, if the frequency response function is calculated from the two accelerometer measurements, then Eq. (13) can be used to verify the attenuation factor determined from the methodology described in Section 1.

Eq. (13) is plotted in Figs 9a(i) and b(i) using experimental data from the Blithfield test rig, when the pipe was excited at Positions 1 and 2 respectively when there was no leak. Figs. 9a(ii) and b(ii) show the corresponding results when a leak was induced. Also plotted is the attenuation calculated using the two methods discussed above, which are given by Eq. (9) and Eq. (11), using data filtered within the frequency regions shown on the graphs. The frequency bandwidth over which the analysis was conducted, was calculated using the method described in [13].

It can be seen that the area method gives a better attenuation estimate when the excitation is set at Position 1, but the half amplitude method gives a slight better attenuation estimate when the actuator is set at Position 2. Although both methods give different 

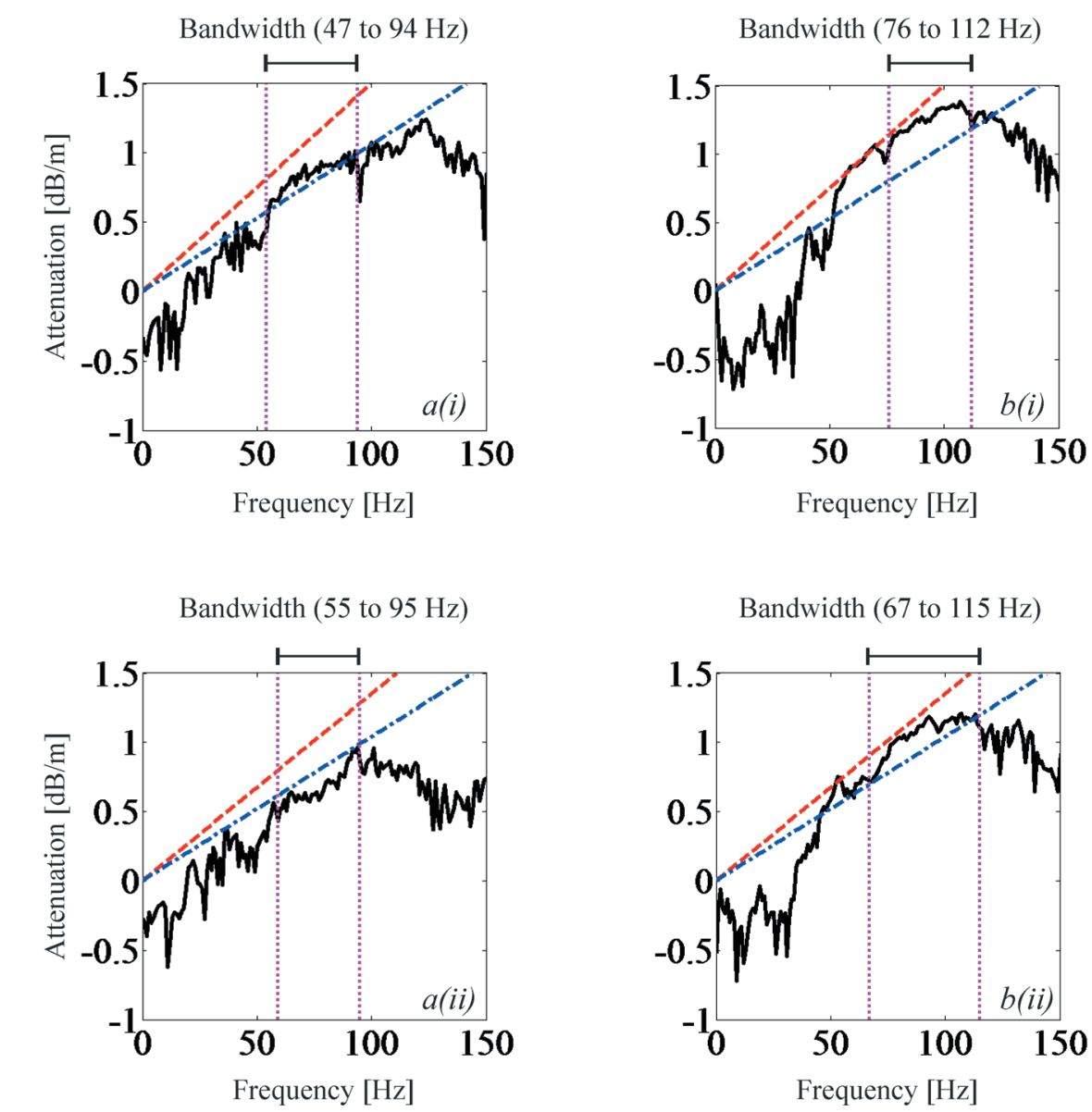
$-=-1=-$ Wave attenuation estimate given by $\mathrm{Eq}$. (9)
-.-. Wave attenuation estimate given by Eq. (11)

Fig. 9. Wave attenuation in $\mathrm{dB} / \mathrm{m}$; a(i) Actuator set at Position 1 with no leak; b(i) Actuator at Position 2 with no leak; a(ii) Actuator at Position 1 with a leak present; $b$ (ii) Actuator set at Position 2 with a leak present

attenuation estimates, in general they give a reasonable attenuation estimate for the cases presented. It can also be seen that the presence of a leak does not interfere dramatically with the estimation of the attenuation factor.

\section{CONCLUSIONS}

In this paper, a method to automatically estimate the wave attenuation factor in buried water pipes, has been proposed. This method involves two measurements made by attaching an actuator to two different positions with co-located accelerometers. The technique is based on the envelope of the crosscorrelation function of the two measurements. An analytical model has been used to describe and to investigate some of the features and limitations of the method. The technique can also be used in the presence of a leak. Experimental results from a bespoke test rig have also been presented to demonstrate the efficacy of the method.

\section{REFERENCES}

[1] Royal, A.C.D., Atkins, P.R., Brennan, M.J., Chapman, D.N., Chen, H., Cohn, A.G., Foo, K.Y., Goddard, K.F., Hayes, R., Hao, T., Lewin, P.L., Metje, N., Muggleton, J.M., Naji, A., Orlando, G., Pennock, S.R., Redfern, M.A., Saul, A.J., Swingler, S.G., Wang, P., Rogers, C.D.F. (2011). Site assessment of multiple-sensor approaches for buried utility detection. International Journal of Geophysics, Article ID 496123, DOI:10.1155/2011/496123.

[2] Cheong, L.C. (1991). Unaccounted-for Water and the Economics of Leak Detection. Water Supply, vol. 9, p. IR 1-1 to 1-6. 
[3] AWWA (1987). Leaks in Water Distribution Systems - A Technical/Economic Overview, American Water Works Association, Denver.

[4] Watergy: Taking Advantage of Untapped Energy and Water Efficiency Opportunities in Municipal Water Systems; from: http://pdf.usaid.gov/pdf_docs/ PNACT993.pdf, accessed on 13/02/2014.

[5] Muggleton, J.M., Brennan, M.J., Pinnington, R.J. (2002). Wavenumber prediction of waves in buried pipes for water leak detection. Journal of Sound and Vibration, vol. 249, no. 5, p. 939-954, DOI:10.1006/ jsvi.2001.3881.

[6] Fuller, C.R., Fahy, F.J. (1982). Characteristics of wave propagation and energy distributions in cylindrical elastic shells filled with fluid. Journal of Sound and Vibration, vol. 81, no. 4, p. 501-518, DOI:10.1016/0022-460X(82)90293-0.

[7] Pinnington, R.J., Briscoe, A.R. (1994). Externally applied sensor for axisymmetric waves in a fluid filled pipe. Journal of Sound and Vibration, vol. 173, no. 4, p. 503-516, DOI:10.1006/jsvi.1994.1243.

[8] Muggleton, J.M., Yan, J. (2013). Wavenumber prediction and measurement of axisymmetric waves in buried fluid-filled pipes: Inclusion of shear coupling at a lubricated pipe/soil interface. Journal of Sound and Vibration, vol. 332, no. 5, p. 1216-1230, DOI:10.1016/j. jsv.2012.10.024.

[9] Muggleton, J.M., Brennan, M.J., Linford, P.W. (2004). Axisymmetric wave propagation in fluid-filled pipes: wavenumber measurements in vacuo and buried pipes. Journal of Sound and Vibration, vol. 270, no. 1-2, p. 171-190, DOI:10.1016/S0022-460X(03)00489-9.

[10] Gao, Y., Brennan, M.J., Joseph, P.F., Muggleton, J.M., Hunaidi, H. (2004). A model of the correlation function of leak noise in buried plastic pipes. Journal of Sound and Vibration, vol. 227, no. 1-2, p. 133-148, DOI:10.1016/j.jsv.2003.08.045.

[11] Muggleton, J.M, Brennan, M.J. (2004). Leak noise propagation and attenuation in submerged plasticwater pipes. Journal of Sound and Vibration, vol. 278, no. 3, p. 527-537, DOI:10.1016/j.jsv.2003.10.052.

[12] Muggleton J.M., Gao, Y., Brennan, M.J., Pinnington, R.J. (2006). A novel sensor for measuring the acoustic pressure in buried plastic water pipes. Journal of Sound and Vibration, vol. 295, no. 3-5, p. 1085-1098, DOI:10.1016/j.jsv.2006.01.032.

[13] Almeida, F.C.L. (2013). Improved Acoustic Methods for Leak Detection in Buried Plastic Water Distribution Pipes. PhD thesis, University of Southampton, Southampton.

[14] Bendat, J.S., Piersol, A.G. (2000). Random Data: Analysis and Measurement Procedures. Third edition, John Wiley \& Sons, Hoboken.

[15] Feldman, M. (2011). Hilbert Transform Applications in Mechanical Vibration. John Wiley \& Sons, Hoboken, DOI: $10.1002 / 9781119991656$ 